\title{
Optical Studies of Crazed Plastic Surfaces ${ }^{1}$
}

\section{Sanford B. Newman and Irvin Wolock}

\begin{abstract}
Optical studies were made of crazed surfaces, principally those of cast polymethyl methacrylate. The techniques used for these studies were multiple-beam interferometry and light and electron microscopy.

The dimensions of the craze cracks produced by short-time stress crazing and by stresssolvent crazing were determined by the above techniques. Stress-crazed specimens contained the smallest cracks observed during this investigation. The dimensions determined in some of these specimens were: Length, 2 to 10 microns; width, 0.1 to 0.25 micron; depth, 0.05 to 0.15 micron. In other specimens and in solvent-crazed surfaces the dimensions were larger. The craze cracks did not appear to approach dimensions unresolvable by the electron microscope, but instead appeared to have a minimum crack length.

An elevation of the surface in the vicinity of craze cracks was observed, of the order of 0.02 micron for cracks produced by stress-crazing and 0.3 micron for those produced by stresssolvent crazing. This rise was observed in crazed specimens while still under load. The typical surface displacement was observed in specimens crazed in tension produced by flexure as well as in pure tension, and was observed in polystyrene and in polymethyl alpha-chloroacrylate as well as in polymethyl methacrylate.

Some crazed specimens in which the crazing had apparently "recovered" when viewed with the unaided eye still exhibited the surface displacement typical of crazed specimens. Experiments conducted by heating stress-crazed specimens and stress-solvent-crazed specimens to accelerate the recovery of crazing indicated that surface recovery is essentially complete in the former case but not in the latter. This difference in recovery behavior is probably due to the nature of the deformation associated with the two types of crazing.

Tensile specimens to which loads had been applied that were slightly too low to produce immediate crazing did not exhibit surface displacements typical of crazed specimens.
\end{abstract}

\section{Introduction}

When transparent thermoplastics such as polymethyl methacrylate or polystyrene are exposed to a sufficiently high tensile stress or to solvents, deterioration of their optical properties occurs. This deterioration results from the formation of cracks that impair light transmission, cause optical aberrations, and reduce the strength properties of the material. Commonly known as "crazing," this phenomenon is encountered in a variety of products ranging from aircraft glazing and canopies to watch crystals, and is obviously of considerable economic importance to manufacturers and users of plastic items. A number of investigators have reported the results of work on the mechanism, characteristics, and elimination or prevention of crazing [ 1 to 7 . $^{2}$ However, crazing remains a serious consideration in plastics applications and many aspects of the problem require intensive study.

Photomicrographs and descriptions of the craze cracks can be found in the literature $[2,4,5]$. The cracks vary greatly in their length and fineness. In tensile specimens crazed by the application of stress or by stress and solvent together, the cracks are almost invariably normal to the applied stress. Individual cracks can usually be observed using a low-power microscope; a $16-\mathrm{mm}$ objective and $10 \times$ ocular is generally more than adequate. The length and number of the cracks can often be determined in this fashion. Some crazed surfaces contain cracks

1 This investigation was conducted at the National Bureau of Standards under the sponsorship and with the financial assistance of the National Advisory Committee for Aeronautics; the help of this agency and members of its staff is gratefully acknowledged.

2 Figures in brackets indicate the literature references at the end of thispaper. that appear to approach the limits of resolution of the microscope, and these require a more powerful tool for the measurement of crack dimension.

Although the length and fineness of crazing cracks can be studied with the bright-field microscope, the determination of the depth of cracks and how they affect surface topography requires other techniques and instruments. Both the electron microscope and the multiple-beam interferometer suggest themselves for these purposes. Saunders [8] has reported observations on minute irregularities in optical surfaces by means of multiple-beam interferometry. Because of the great magnification of minute detail and the relatively large surface areas that can be examined simultaneously, multiple-beam interferometry lends itself readily to the precise study of the topography of plastic sheets. The multiple-beam or FabryPerot-type fringe is a classical interferometric technique. A number of applications and refinements of the method have appeared in recent years, many of which have been the work of Tolansky and his associates $[9,10]$. These techniques yield magnification of detail in one direction only, the direction of height or depth. In the measurement of height this method equals or surpasses results obtained with the electron microscope, at the same time eliminating the elaborate specimen preparation required by the latter instrument. The electron microscope, however, yields other information that would be difficult or impossible to obtain with available interferometric techniques. Some indication of how these tools supplement one another is evident in the observations presented.

The acrylic and styrene plastics used in this study are amorphous high polymers. In these materials the total response to an applied stress contains three 
components. These are the instantaneous elasticity, retarded configurational elasticity, and flow. When the stress is removed there results an instantaneous recovery of the deformation due to the first component and a slower recovery from the second. Deformation due to flow is not recovered. These elements in the viscoelastic behavior of the polymer can be related to observed microstructural characteristics of crazed surfaces. It also aids in categorizing the mechanisms responsible for changes in the cracks and their associated deformations on exposure to elevated temperature or stress.

\section{Materials}

Specimens of cast commercial polymethyl methacrylate were the ones chiefly used in this investigation. Both the general-purpose and heat-resistant grades were studied. The sheets of cast polystyrene were also of commercial origin. The cast polymethyl alpha-chloroacrylate was experimental polymer.

Samples of acrylic laminate were also examined. The two outer layers of this laminate consisted of polymethyl methacrylate and the interlayer was plasticized polyvinyl butyral. The inner face of the acrylic outerlayer was examined.

\section{Methods}

\subsection{Preparation of Crazed Specimens}

The standard tensile specimens used were type I specimens described in Method 1011 of Federal Specification L-P-406b [11]. The specimens were stress-crazed by tensile loading in a hydraulic universal testing machine with the rate of head separation at $0.05 \mathrm{in} . \mathrm{min}$. In some cases, the specimens were loaded until crazing occurred and then the load was released, whereas in other cases the load was applied until failure occurred.

This same type of specimen was also stress-solvent crazed. In this case, a sufficiently high load was applied and then the surface of the specimen was exposed to solvent either by holding a benzenesaturated blotter against one face for $10 \mathrm{sec}$ or by stroking the surface with a small camel's hair brush saturated with solvent.

Whereas crazing in polymethyl methacrylate manifests itself primarily on the surface, in cast polystyrene and cast polymethyl alpha-chloroacrylate sheets it is also apparent throughout the matrix. In the two latter materials, the interior craze cracks appear to be associated with impurities. However, the surface craze cracks of these two materials were similar in shape and orientation to those observed in polymethyl methacrylate.

Flow lines were observed in some crazed specimens of polymethyl methacrylate, emanating from the ends of craze cracks. These flow lines were observed after the craze cracks appeared, and formed at angles of $35^{\circ}$ to $45^{\circ}$ to the cracks. The craze cracks did not appear to grow after the flow lines appeared.
Tapered tensile specimens which had been stresssolvent-crazed for use in another investigation were also examined. These specimens tapered in width from 0.500 to 0.333 in. over a 3 -in. reduced section. The methods of crazing were the same as those described in the previous paragraph.

Solvent-crazed surfaces were also prepared by placing 2-in. squares cut from cast sheets of polymethyl methacrylate in boiling water for 2 or 3 min and then exposing them to monomeric methyl methacrylate until they were visibly crazed.

Flexural specimens were stressed statically as cantilevers to produce crazing.

\subsection{Preparation of Surfaces for Interferometric Studies}

Exposed surfaces of the plastic sheets quickly accumulated a film of dust particles from the surrounding air. These particles were thoroughly removed to facilitate the microscopic examination and permit the deposition of suitable metallic films on those specimens used for multiple-beam interferometry. Cleansing was carried on with extreme caution, as even gentle wiping with commercial cellulose tissues or with absorbent cotton was sufficient to produce surface marks that could be detected microscopically. Immersion in a stream of tap water followed by blotting with a good grade of lens tissue caused little apparent surface degradation. Such a procedure, however, is not adequate for removing all detritus, and foreign bodies were constantly encountered during the examinations.

The glass optical flats and other reference surfaces were handled by washing in soap and water and drying with lens tissue or boiled cotton or linen cloths. Old silver coatings were removed with a 30 -percent solution of hydrogen peroxide.

The cleansed specimens and reference surfaces for multiple-beam interferometric studies were then placed in a metal evaporator. A film of silver was deposited on the crazed and uncrazed surfaces to be examined and on the optical or other glass surface to be used as the reference flat.

Plastic specimens on which the fringes were observed in transmission were coated with a laver of silver 400 to $500 \mathrm{~A}$ in thickness. Such films approach a reflectivity of 90 percent. Tolansky [9] presents considerable detail of the technique for evaporating. a layer of the proper thickness and reflectivity. The films applied to these specimens were formed in a vacuum of $1 \times 10^{-4} \mathrm{~mm} \mathrm{Hg}$ or better. The heated tungsten filament was held at least $20 \mathrm{~cm}$ from the plastic to avoid overheating the specimen. A film of the desired thickness range was a deep blue color when viewed in transmission. With experience it was possible to make a visual estimate of the film thickness by its color and transmission. Observing the image of a small piece of white paper placed beneath the specimen also assisted in evaluating the layer of metal during its deposition. The extinction of the image can be used as the end point. Alternately, a measured amount of silver computed 
to be sufficient to produce a film of the desired thickness at the distance of the specimen from the filament can be evaporated in its entirety.

Silver films of the same thickness were evaporated on all reference surfaces.

A layer of silver sufficiently thick to be totally reflecting (at least $1000 \mathrm{~A}$ ) [12] was deposited on the plastic surfaces used for multiple-beam interferometric studies by reflection.

\subsection{Preparation of Replicas for Electron Microscope Studies}

Specimens were prepared by a slight modification of a replica technique described by Zworykin and Ramberg [13]. Crazed specimens of polymethyl methacrylate were placed in a vacuum unit which was pumped to $1 \times 10^{-4} \mathrm{~mm}$ of $\mathrm{Hg}$ or less. Silver was evaporated perpendicularly on the specimens from a distance of $20 \mathrm{~cm}$. A totally reflecting surface of silver was obtained (thickness of $1000 \mathrm{~A}$ or more). The specimens with their evaporated coatings were removed from the unit and melted paraffin was applied to the edges of the surface bearing the silver layer. The coated plastic was then placed in an electroplating solution and a layer of copper about 0.01 in. thick was deposited on the back of the silver film. The solution contained $4 \mathrm{~g}$ of copper sulfate and $0.6 \mathrm{mg}$ of sulfuric acid per $20 \mathrm{ml}$ of water. Deposition of the copper was accomplished with 0.1 to $0.2 \mathrm{amp} /$ in. $^{2}$, but this value can be varied over wide limits. The metal layer was then cleanly stripped from the plastic specimen. The paraffin prevents the electroplating solution from attacking the evaporated film at the edges and eliminates the right-angle fins that tend to grow over the edge of the plastic during plating, thus complicating removal of the metal. The metal replica was washed in distilled water and dried.

The replicas were shadowed with chromium while displaced $4 \mathrm{~cm}$ vertically and $16 \mathrm{~cm}$ horizontally from the heated filament containing the metal. A thin layer of collodion in amyl acetate (about a 1\% solution) was flowed over the replica, the excess allowed to drain, and the metal replica permitted to dry in a dust-free location. Then a 25-percent (by volume) solution of nitric acid was used to dissolve away the copper and silver, leaving a positive shadowed replica of collodion. The replica was washed in several changes of distilled water and mounted on wire grids. Steps involved in replication are shown scbematically in figure 1.

\subsection{Microscopic Techniques}

For bright-field observations, the crazed specimens were examined with a compound microscope equipped with $16-\mathrm{mm}$ or $4-\mathrm{mm}$ objectives and a $10 \times$ ocular. Some tensile specimens were also studied with a dark-contrast 4-mm phase contrast objective. However, it was found that the methods of multiplebeam interference we 'e most valuable for determining structures present in the plastic surfaces.

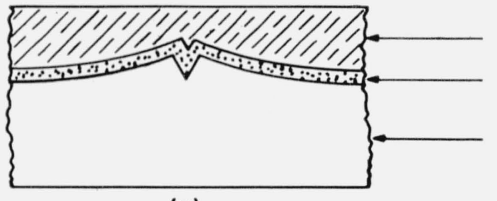

COPPER LAYER

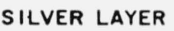

(a)

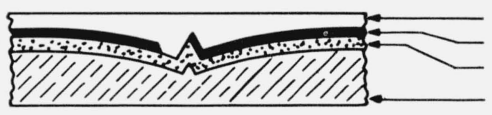

COLLODION FILM CHROMIUM FILM SILVER LAYER

(b) COPPER LAYER

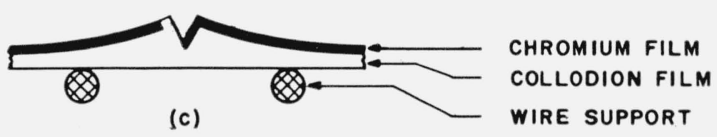

Figure 1. Preparation of electron microscope specimens.

(a) Silver is vacuum-deposited on the plastic sheet. A thicker layer of copper is electrodeposited on the silver. (b) The silver and copper layer is stripped from the plastic and shadowed with chromium. Collodion is then flowed over the chromium, forming a film replica. (c) Silver and copper films are removed with acid and the chromium shadow is mounted on a supporting grid.

\section{a. Multiple-Beam Interferometry}

(1) Theory. Observations with multiple-beam interferometry augmented the details revealed by transmission microscopy. The interference fringes formed in the thin film of air between a reference surface (optical flat) and the surface of a plastic sheet are governed by the well-known expression $n \lambda=2 t \cos \theta \mu$, where $n$ is the order of interference with a film of thickness $t, \lambda$ the wave length of the light used, $\mu$ the refractive index of the air layer, and $\theta$ the angle of light incidence. When fringes are formed between unsilvered surfaces, only two light beams interfere. The lines of maximum intensity in such fringes are often difficult to determine and precise topographical detail cannot be measured. Multiple beams are produced by coating the surfaces producing interference with a highly reflecting, thin film of silver. The absorption of the film is held to the minimum possible at reflecting coefficients of 0.7 to 0.9 . The successive reflecting of the beams between the silvered surfaces is sufficient to influence the resulting interference. This leads to a great increase in precision through sharpening of the fringes when all the beams are collected at the focus of a lens. The theory of interference by multiple beams is discussed in detail by Ditchburn [14] and Tolansky [9].

The equivalent positions of the fringes in the interferograms mark the loci of points separated by changes in the thickness of the interferometer air wedge equal to $\lambda / 2$. Throughout this investigation $\lambda=546 \mathrm{~m} \mu$. The interferogram, then, is a series of lines or fringes that reflect the surface contours of the specimen surface. When the fringes are straight parallel lines, the specimen surface approximates the flatness of the reference surface with which it is compared. Changes in the distance 
between these lines are due not only to changes in microscopic magnification but are also dependent on the wedge angle between specimen and reference surface. Because the reference surface may be considered perfectly plane over the limited areas in a single field, all deviations in parallelism or direction of the fringes are a measure of the change in height of the specimen surface along the length of the fringe. Because of the fringe sharpness and the known geometric relations between fringe and surface contour, the specimen surface contour can often be determined with a high degree of accuracy.

For example, if we consider the interferogram in figure 7 and indicate the significant features by letters, the method of analysis will be obvious. The craze crack, which in this case is not a straight line, is indicated by XY. Because the linear distance from fringe I to fringe II also represents a change in the air wedge height of $\lambda / 2$ or $273 \mathrm{~m} \mu$, then the surface containing point $\mathrm{B}$ has risen, or fallen, approximately this amount in the distance AB. Similarly, the surface containing D has risen (or fallen) approximately four-fifths of $273 \mathrm{~m} \mu$ in the distance CD, and point $\mathrm{F}$ about one-third of $273 \mathrm{~m} \mu$ in the distance EF. The fringes, then, yield a contour map of the surface topography wherever the fringes are propagated. In figure 7 it is obvious that the changes in surface height occur close to the craze crack itself and appear to decrease at the end of the crack length. Readjustment of the reference surface and the wedge angle will displace or change the fringe pattern, permitting the acquisition of more detailed information or of measurements at other points on the surface. The fringe separation will affect both sensitivity and precision of the measurements.

From the interferogram alone it is impossible to determine whether a discontinuity in the surface is a height or a depth. This can be ascertained, however, by pressing lightly against the plates of the interferometer. The fringes in this interferometer move in the direction of the thicker part of the air wedge. The considerations involved are shown schematically in figure 2. Other methods are avail-

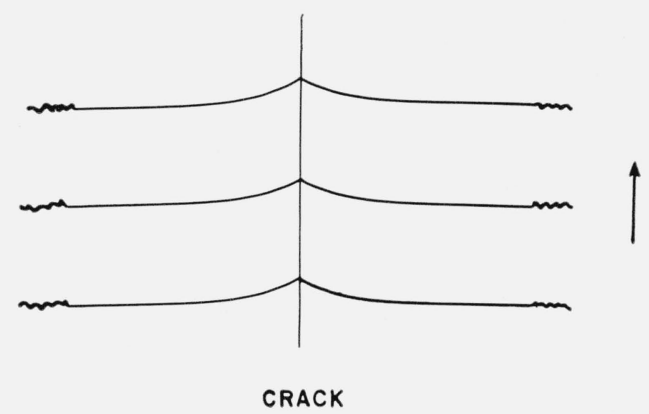

Figure 2. Height or depth indication in interferograms when wedge angle is changed.

If the wedge angle is decreased, the fringes move toward the wedge opening, indicating direction of decreasing distance between surfaces. This is the direction of the arrow. 'Thus the humps in the fringes are heights. able and were used for checking the direction of surface discontinuities. For example, a step wedge consisting of a thin layer of plastic is applied to part of the surface. Where the fringes reach the edge of the film they are deflected in direction by the thickness of the plastic film, thus indicating the direction for a height. Brossel [15] has described an extended source method of determining fringe direction which is easily applied to the specimens.

(2) Transmission. Specimens with semireflecting films of silver were used. An inverted universal microscope was modified to accept an $\mathrm{AH}-4$ highpressure mercury arc lamp in the transmissionsource housing. The specimen was mounted in the microscope as shown in figure $3 \mathrm{~b}$. The parallel beam from the mercury lamp passes through a filter combination (Wratten 55 and Corning didymium) and enters the interference system, consisting of a semireflecting specimen and a semireflecting reference surface. The metal films were face to face. The microscope was focused on the fringes formed between the silvered surfaces of the specimen and the reference plate.
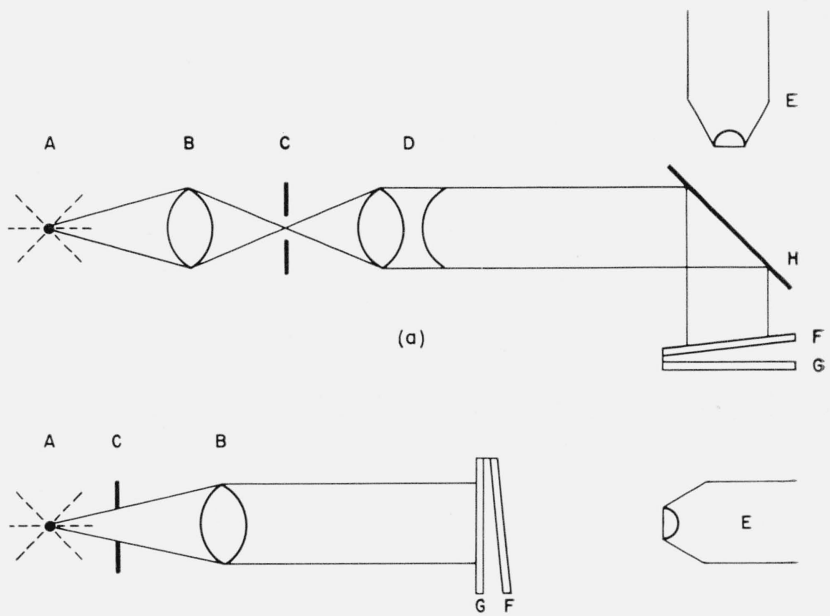

(b)

Figure 3. Optical arrangement for multiple-beam interference.

(a), Reflection; (b), transmission. A, Light source; B, condenser; C, slit; D, collimator; E, objective; F, specimen; G, reference surface; H, beam splitter.

(3) Reflection. Specimens with totally reflecting silver films were used. The same universal microscope was used as with the transmission method described above. However, in this case the highpressure mercury lamp was inserted in the reflectionsource housing and the specimen viewed as shown in figure 3a. The parallel beam of the lamp was filtered, and fell on the interference system of the totally reflecting specimen and the partially reflecting reference plate from the semireflector. The microscope was focused on the fringe system between the specimen and the reference surface. 


\section{b. Electron Microscopy}

The electron microscope used was a $50-\mathrm{kv}$, permanent-magnet table model.

\section{Observations and Discussion}

\subsection{Dimensions of Craze Cracks}

Many of the individual cracks in the crazed specimens were easily distinguished by bright-field microscopy. Cracks observed by this technique are shown in the work reported by Russell [5] and others $[2,3,4]$. This does not mean that all cracks, particularly those in "blushed" or finely crazed areas, can be well resolved by this method. Chromiumshadowed collodion replicas were prepared from the surfaces of specimens of cast polymethyl methacrylate that had been crazed in short-time tensile tests. Specimens were chosen that had exceptionally fine cracks in the crazed areas. Figure 4 is an electron micrograph of one of these replicas. Despite the number of steps involved in its production, these replicas provide good resolution, and the craze crack appears as a height with the evaporated metal piled up on the side nearest the filament (black line) and

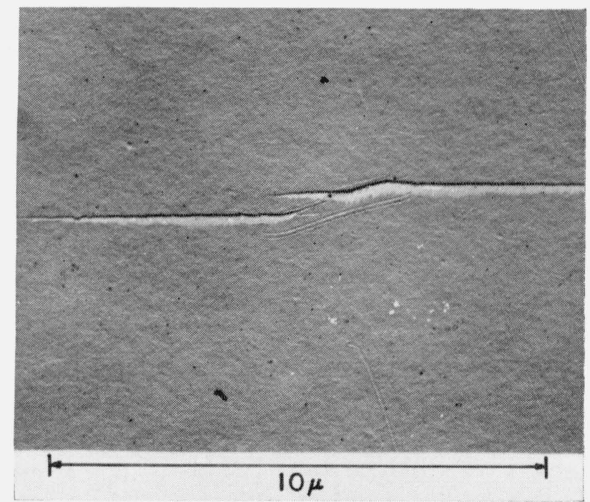

Figure 4. Electron micrograph of a crack in a stress-crazed surface of polymethyl methacrylate.

Collodion replica, chromium shadowed 4:1.

the light shadow appearing as a white or gray line. It is possible to make some accurate determinations of the dimensions from these replicas since the depth of the crack is given by the shadow, the maximum half-width by the black line of piled-up chromium, and the length directly from the impression. The craze cracks examined in the electron microscope varied in length from 2 to $10 \mu$.

Their widths ranged from 0.10 to $0.25 \mu$ and their depths from 0.05 to $0.15 \mu$. In no case did the cracks appear to approach dimensions unresolvable by the electron microscope. The smallest cracks were also much larger than the structure observed in the replica substrate. This implies a minimum craze-crack length. One possible interpretation is that a certain minimum amount of energy is required to cause separation of the chain segments leading to crazing, and this energy threshold results in a minimum crack size. Alternately, there could exist a grain structure analogous to that of metals, which determines the threshold crack size. Further studies will be made to determine exactly the minimum crack size under various conditions.

\subsection{Surface Dislocations Associated With Cracks}

Uncrazed plastic surfaces yielded interferograms of the type shown in figure 5. Commercial samples of polymethyl methacrylate showed some fine detail along the fringes but no gross details other than those attributable to contamination or body deformation.

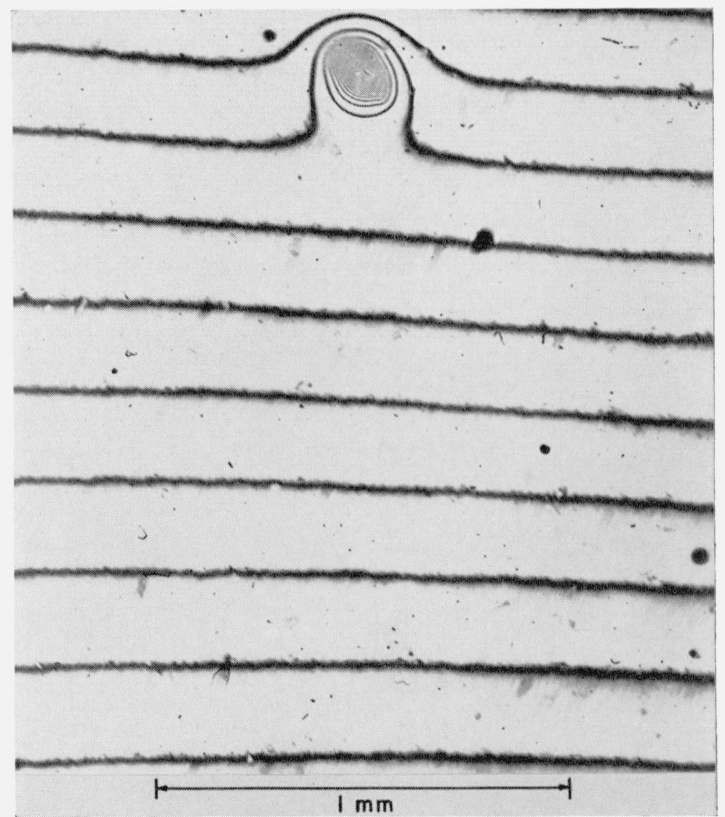

Figure 5. Multiple-beam interferogram of an uncrazed surface of commercial cast polymethyl methacrylate.

Some fine detail is observable along the fringes, and body deformations also exert a measurable effect on the over-all fringe pattern. The rounded detail is typical and apparently due to a mount of adhesive from the masking paper. Source is the $546-\mathrm{m} \mu$ line of a mercury lamp.

Interferograms of crazed surfaces, whether stresscrazed, solvent-crazed, or stress-solvent-crazed, exhibited fundamentally the same surface topography in the region of the craze cracks (figs. $6,7,8$ ). The individual cracks could be seen to occupy an area that is displaced from the rest of the surface. This displacement appeared to increase with the size of the crack. It followed the crack very closely, as can be seen in the interferogram of the unoriented solventproduced cracks (fig. 7).

The surface dislocations caused by the craze cracks varied considerably in magnitude, not only from crack to crack but along the length of the crack as well. Because the fringes can be best resolved by very 


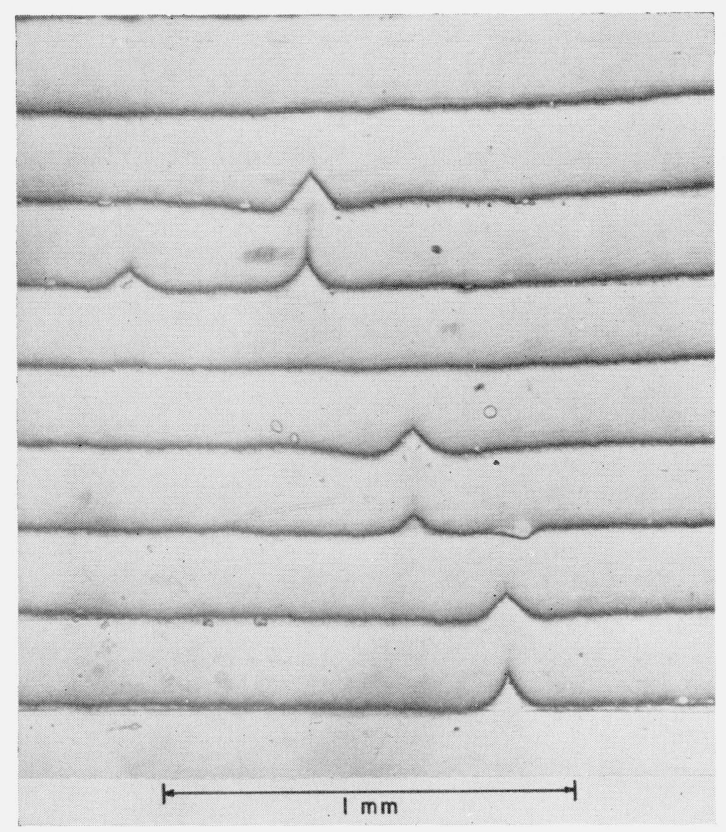

Figure 6. Multiple-beam interferogram of the surface of a sheet of polymethyl methacrylate crazed in flexure.

The discontinuities in the parellel fringes show the loci of the cracks which themselves are practically invisible. Source is the $546-\mathrm{m} \mu$ line of a mercury lamp.

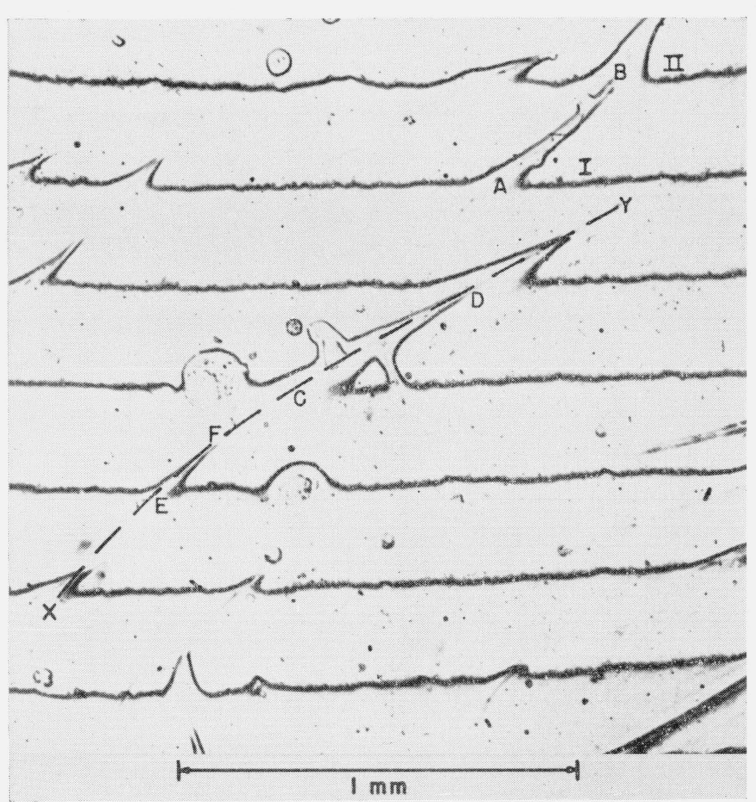

Figure 7. Multiple-beam interferogram of the surface of a sheet of polymethyl methacrylate crazed by exposure to solvent.

The numerous sharp discontinuities in the fringes mark the loci of the craze cracks which themselves are practically invisible. Source is the $546-\mathrm{m} \mu$ line of a mercury lamp.

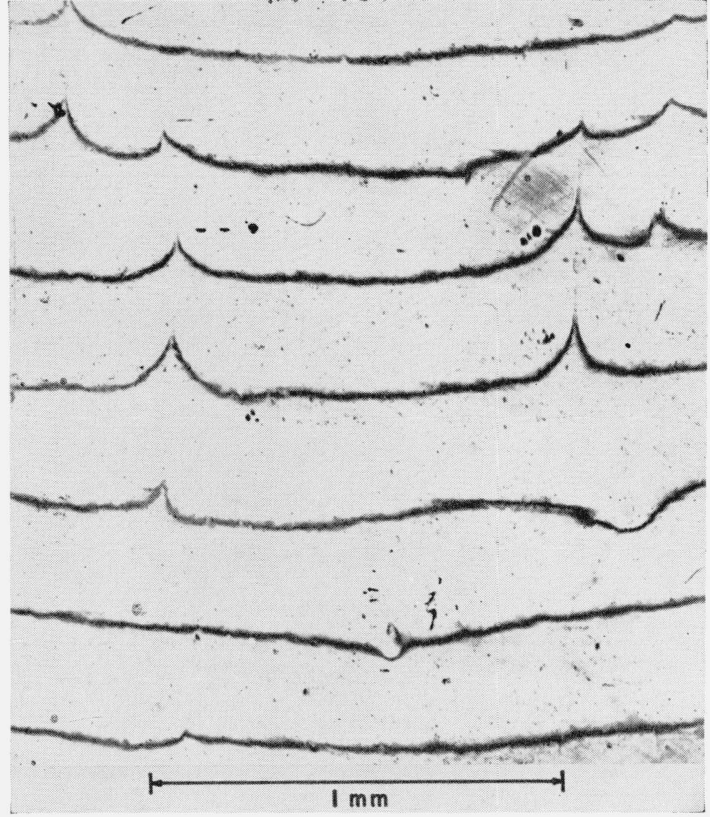

Figure 8. Multiple-beam interferogram of the surface of a sheet of polymethyl methacrylate crazed by application of stress and solvent.

The sharp discontinuities in the fringes mark the loci of the craze cracks. Because the specimen was stressed to failure, the long period fringe contours are the result of body deformation. Source is the $546-\mathrm{m} \mu$ line of a mercury lamp.

low-powered objectives in the simple technique previously described, the very fine cracks are somewhat difficult to study quantitatively, but the longer, coarser cracks are easily measured. For this reason, the stress-solvent-crazed material provides better demonstrations of the observations than the stresscrazed specimens.

Dimensions of the details of one of the cracks shown in the interferogram in figure 8 are shown schematically in figure 9. Although, as indicated previously, there is considerable variation, the maximum change in height for areas around stress-solventproduced cracks was found to be about $1 / 2 \lambda$ or approximately $270 \mathrm{~m} \mu$. For stress-produced craze cracks the elevation is much less, apparently only a few hundred angstroms.

By using the techniques previously described, it was shown that the area around each crack was elevated and not depressed. The phase-contrast microscope was also used for this purpose. With a darkcontrast diffraction plate in the objective, the part of the specimen having the greater optical path will be darker than the surrounding parts having shorter paths. Thus, the crack itself appears bright, and the thickening adjacent to it is darker than the surround.

The behavior of the plastic sheet in forming these surface discontinuities around each crack can be simulated in the following experiment with a sheet 

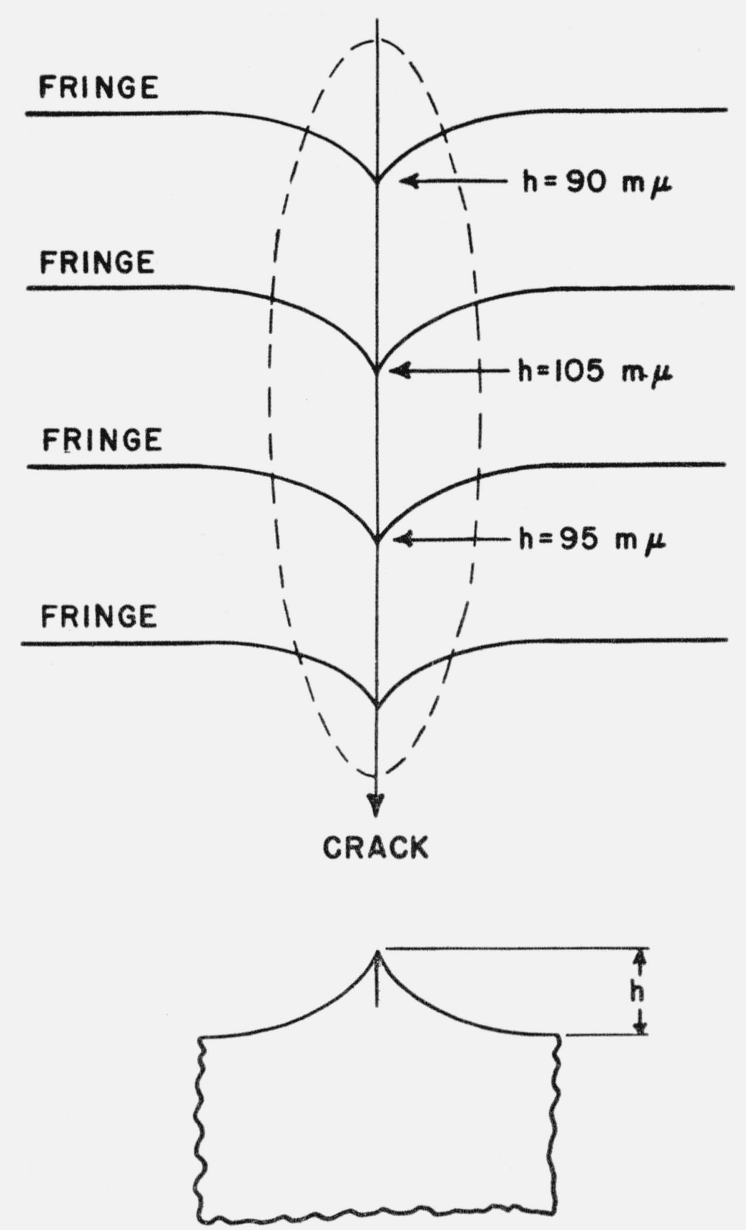

\section{SECTION THROUGH CRACK}

FiguRE 9. Schematic representation of a craze crack from figure 8 (slightly idealized).

The encircled portion shows the surface area affected $\times 100$.

of rubber. A 1/16-in.-thick strip of rubber was superficially notched on one face with a sharp razor blade, the notch not extending to the edges, and with its long axis normal to the major axis of the specimen strip. When a tensile load, sufficient to cause considerable elongation, was applied parallel to the specimen's major axis, a raised area appeared around the notch. On releasing the tension the raised area subsided. When the specimen was observed on the unnotched side just opposite the notch while the tension was applied, it could be seen that a concavity formed at this point. The material of the rubber strip was apparently pulled up around the lips of the cracks at the expense of material from the opposite side. The rubber, being above the glass transition at room temperature, recovered on removal of the load, there being no delayed elasticity or flow. Recovery in the plastic sheets, which were below the glass transition, was much slower and incomplete because of these viscoelastic properties.
A complete explanation of this phenomenon of surface dislocation would require a lengthy and highly detailed stress analysis. Qualitatively, it can be said that the center axis through the cross section of the specimen is displaced locally by the formation of a craze crack due to the decrease in thickness at the point of the crack. The center axis moves to realine with the load line, and material on either side of the crack is displaced away from the center axis. In the case of the rubber sheet, this realinement also produces a concavity in the surface opposite the notch. However, the concavity observed on the reverse side of the rubber specimen could not be detected in the crazed plastic. Crazing: is a phenomenon that for practical considerations is restricted to a thin surface membrane. Both the high modulus of elasticity and the thickness of the plastic specimen hinder the formation of the concavity observed in the rubber strip.

\subsection{Surface Dislocations Under Load}

The typical fringe contour showing a rise in the surface in the vicinity of craze cracks can be shown to be present while the crazed specimen is still under load. A series of observations was made on one stress-crazed and one stress-solvent-crazed specimen while still under load. Each specimen was clamped in a specially designed jig, which could be placed on the microscope stage and which would permit the application of a load to the specimen by means of a bolt at one end. An unmeasured load was applied for several months to the stress-crazed specimen. The stress-solvent-crazed specimen was under load for several weeks prior to the application of solvent to the surface. With the specimen still under load, the silver film was applied in vacuum and the specimen examined in the interferometer. Under load, the contour fringes indicated much the same type of displacement as in the other crazed specimens. For the stress-solvent-crazed specimen (fig. 10), the fringes were somewhat exaggerated when compared to specimens solvent-crazed in short-time loading. It is doubtful that the increased deformation was due to the fact that the specimen was still under stress, because on the removal of the load there was little, if any, recovery. Increasing the duration of the loading increased the size of the surface discontinuities. It can be seen from figure 10 that there are portions of the craze cracks that rise more steeply than the remainder, as if the lips of the cracks had yielded locally.

\subsection{Recovery in Crazed Surfaces}

Some recovery of crazing undoubtedly does occur in crazed specimens over long periods of time after the stress is removed. Crazed specimens of polymethyl methacrylate that were stored at room temperature for several months were found to have lost all or much of the visual evidence of crazing. On microscopic examination some residual evidence of 


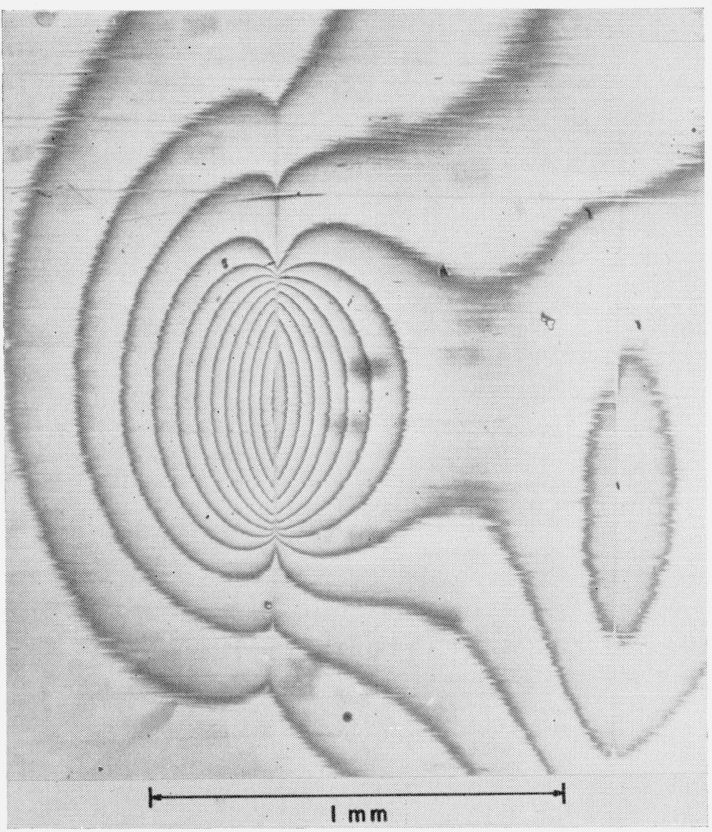

Figure 10. Multiple-beam interferogram of the surface of a sheet of polymethyl methacrylate crazed by application of stress and solvent.

The stress has not been removed. Horizontal lines intersecting the fringes were caused by abrasion resulting from wiping the surface with lens tissue. Source is the $546-\mathrm{m} \mu$ line of a mercury lamp

crazing could be detected. A number of tensile specimens that had been stress-crazed some months earlier were silvered and examined in the multiplebeam interferometer. Although these specimens appeared uncrazed to the unaided eye and even in the bright-field microscope, the sites of the craze cracks were easily distinguished by multiple-beam interferometry. This residual crazing was evidently too slight to affect the optical properties of the sheet sufficiently to reveal the crazed condition without the aid of optical tools.

Some further observations on a number of crazed plastic specimens that had been heated, indicated the general aspects of this optical recovery. Tensile specimens of various types of commercial polymethyl methacrylate sheet were stress-crazed in short-time tensile tests. One of the halves of each specimen was heated for various periods of time up to 15 hours at $150^{\circ} \mathrm{C}$ in an air-circulating oven. When examined with the bright-field microscope, the half of the specimen that was not heated showed the typical cracks oriented with their long axis, normal to the principal stress (fig. 11). However, the half that had been heated appeared clear and uncrazed. Multiplebeam interferometry of the heated portion also revealed no indication of the surface dislocations associated with crazing.

Somewhat different results were obtained with specimens that were crazed by exposure to solvent while under stress. After crazing, loading was continued until failure occurred. Half of each broken specimen was given the same heat treatment as described for the stress-crazed plastic. The heated

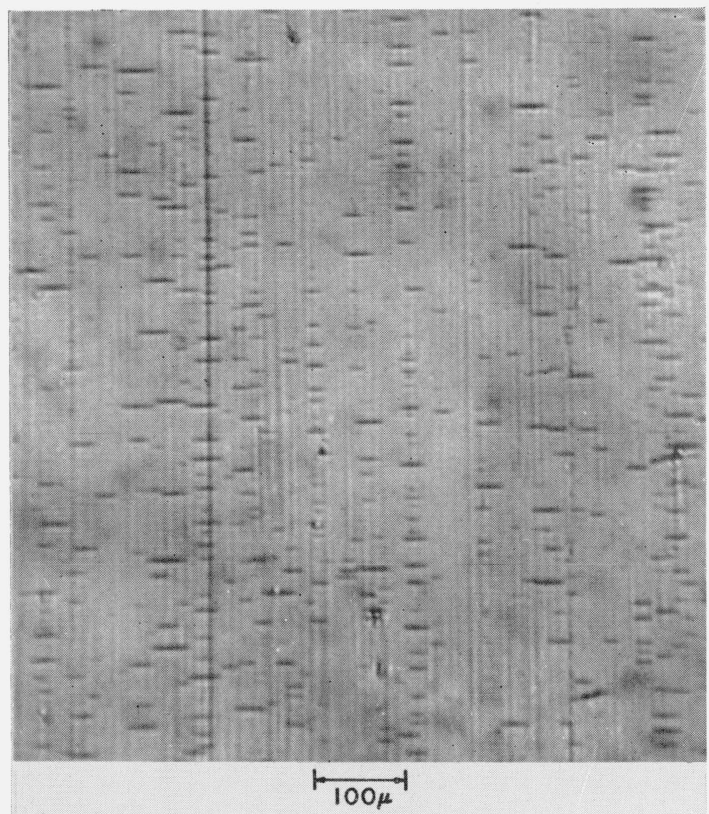

Figure 11. Surface of stress-crazed polymethyl methacrylate. The stress was applied normal to the long axis of the cracks. Vertical lines are the result of wiping the surface with lens tissues.

specimens again showed no evidence of crazing when viewed with the bright-field microscope. Multiplebeam interferometry of the heated portion, however, revealed surface dislocations similar to those observed on unheated stress-solvent-crazed specimens. These dislocations were more rounded and gradual in contour and lacked, for the most part, the abrupt discontinuity at the crack (fig. 12). In addition, there

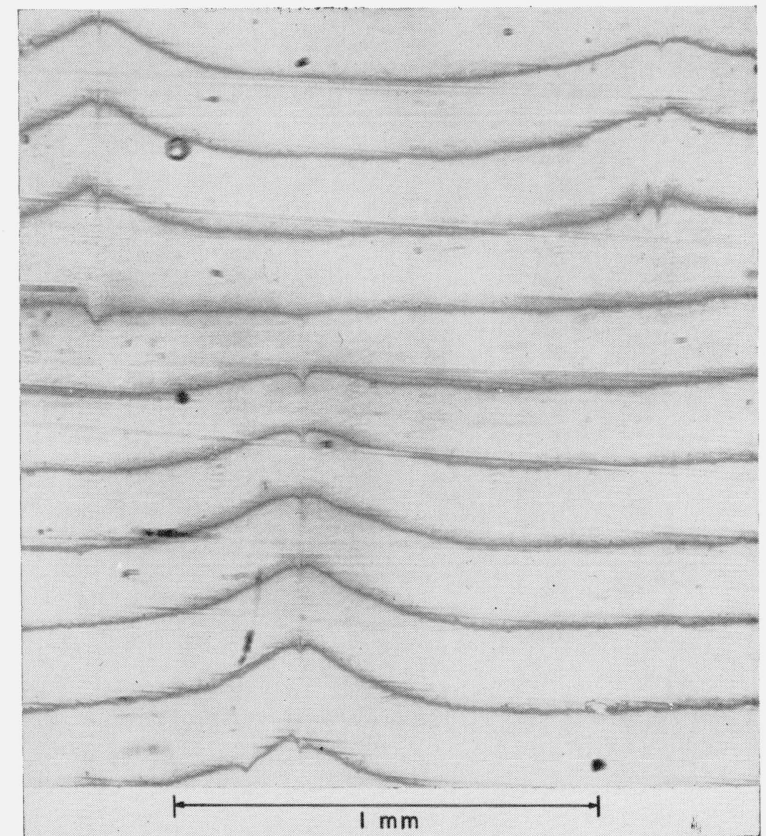

Figure 12. Multiple-beam interferogram of the surface of a stress-solvent-crazed specimen of polymethyl methacrylate sheet after heating to $150^{\circ} \mathrm{C}$.

Source is the $546-\mathrm{m} \mu$ line of a mercury lamp. 
appeared to be a widening of the crack itself, forming a trough along the crest of the dislocation (cf. fig. 8).

These experiments indicate that when the crazed surface is heated above the glass transition temperature, the surface tends to flow. Because the crack is largely responsible for the changes in optical path of the transmitted light, any change eliminating these physical discontinuities would improve the optical properties of the sheet. The slight and gradual thickenings of the sheet caused by the dislocations around the crack would be expected to impair the optical properties of the crack in less obvious fashion. In the stress-crazed surfaces the flow at $150^{\circ} \mathrm{C}$ is sufficient to erase all evidences of crazing in a relatively short period of time. At room temperature, the recovery is much slower. Stress-solvent craze cracks also tend to "recover", but the recovery is incomplete. The discontinuity of the crack is largely obliterated and the sharp edges are rounded. Some relaxation of the edges of the crack also occurs, forming the trough in the crest. Flow is less effective in eradicating the comparatively broad surface dislocations surrounding the crack.

The difference in behavior for the two types of crazing is probably due to the nature of the deformations associated with each type. In the case of stress-crazing in short-time tensile tests, a "frozenin" elastic deformation is apparently involved, which would be completely recoverable. In stress-solvent crazing, however, considerable flow, at or near the surface, probably occurs, due to the plasticizing action of the solvent. This deformation is permanent and complete recovery is not observed as a result of heating.

In view of the great sensitivity of the multiplebeam technique in revealing evidence of crazing, experiments were conducted to determine if crazing could be detected by this method prior to its observation by the unaided eye. In the standard tensile tests described previously, specimens of cast polymethyl methacrylate crazed at strains of slightly more than 3 percent. Tensile specimens were strained, therefore, to only 1, 2, and 3 percent, and the load removed. These specimens, which appeared uncrazed to the eye, exhibited no craze cracks when observed by multiple-beam interferometry and phase or bright-field microscopy. Similarly, unstressed polymethyl methacrylate sheet showed no crazing when examined by these sensitive techniques. These results indicate that the observation of the onset of crazing by the eye is an adequately sensitive technique.

\subsection{Loci of Craze Cracks}

Distribution of the cracks across the section of the specimens differed with the polymer involved. All of the craze cracks observed in polymethyl methacrylate involved the surface, that is, the surface plane intersected the cracks. While some surface crazing was observed in polystyrene and in polymethyl alpha-chloroacrylate, there was also considerable internal crazing, i. e., cracks that did not_open on the surface. However, the surface craze cracks of the polystyrene and the polymethyl alpha-chloroacrylate were similar in shape to those of polymethyl methacrylate.

Internal cracks in the polystyrene specimens were distributed fairly homogeneously throughout the section. Cracks would occur in groups of two or more, and in most instances the group was associated with a visible particle of foreign matter (fig. 13). It

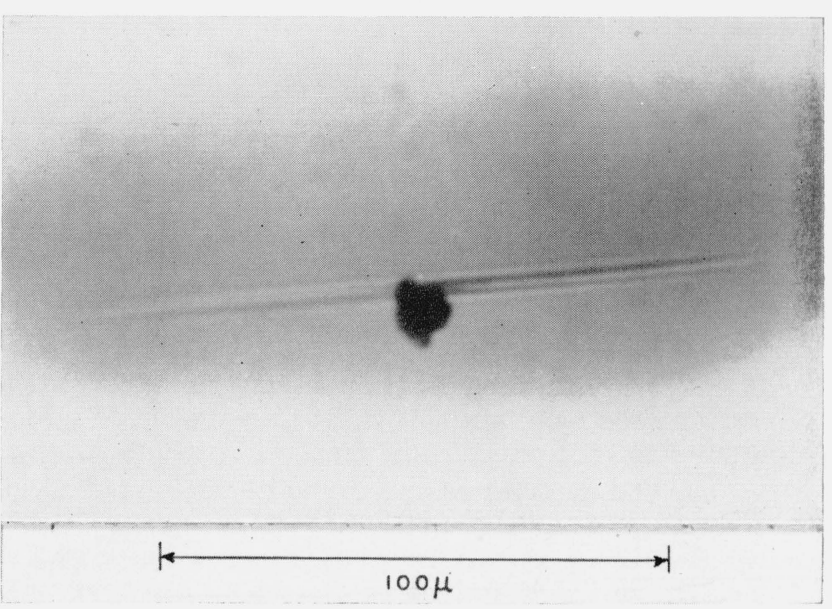

Figure 13. Crazing in polystyrene.

A single craze crack associated with a visible inhomogeneity.

would appear that internal crazing appeared in the polystyrene specimens at physical discontinuities, such as fragments of impurities, which were visible, or minute areas of inhomogeneities, which were not visible.

Crazing in the polymethyl alpha-chloroacrylate was mainly restricted to the center of the specimen section. Just under each cast surface was a clear section containing few, if any, cracks as shown in figure 14. When viewed with a microscope equipped with a double diaphragm [16], the cracks could be

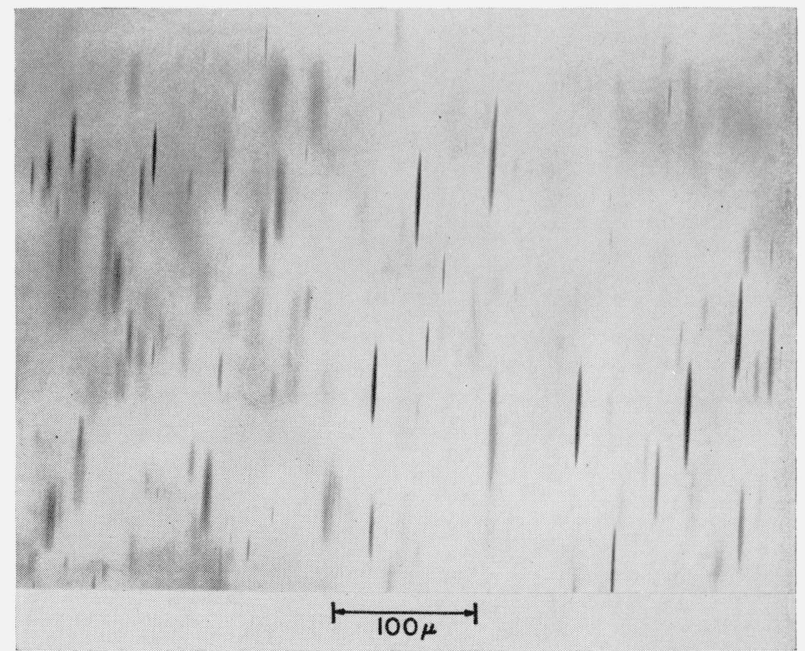

FIgURE 14. Crazing in polymethyl alpha-chloroacrylate.

The cast surface of the specimen is at the top of the photograph; the interior of the specimen is viewed through a machined and polished edge of the specimen. Note the clear area immediately adjacent to the edge. 
seen to be areas of much lower index than the surrounding matrix. This would indicate that the cracks may be voids in the resin.

It is interesting to observe that the crazed specimens of polymethyl alpha-chloroacrylate, with a clear area near each surface, bear a striking resemblance to specimens of polymethyl methacrylate that had been subjected to atomic radiation in a pile and heated [17]. In the latter case, bubbles were formed in the interior of the specimens with a clear section extending inwards approximately $1 \mathrm{~mm}$ from all external surfaces. The authors explained that the bubbles in the interior of the material are due to gases that are evolved in the degradation of polymethyl methacrylate by irradiation. Bubbles do not occur near the surface because the gases produced there can escape.

\subsection{Flow Associated With Cracks}

During the studies of crazed surfaces with the optical microscope, a network of diagonal lines which appeared to emanate from the ends of craze cracks was observed in some stress-crazed tensile specimens of polymethyl methacrylate. The lines are more easily seen and photographed if the microscope is focused below the surface of the specimen (fig. 15). The lines were not observed on all crazed specimens of polymethyl methacrylate, even though the conditions of crazing were duplicated in replicate specimens. The angle between the trace of these flow lines on the surface and the craze cracks was somewhat variable. Values from about $35^{\circ}$ to $45^{\circ}$ were obtained on different specimens (fig. 15). Flow lines were also noted in one of the crazed specimens which had apparently "recovered" when examined without optical aids.

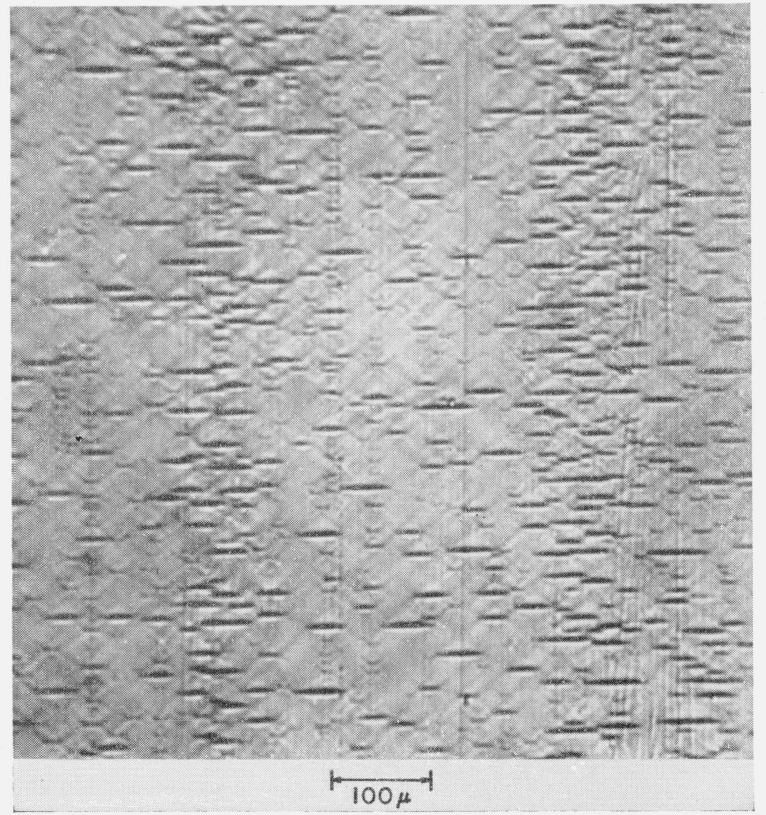

FIgURE 15. Surface of a stress-crazed tensile specimen of polymethyl methacrylate.

The image has been slightly defocused to emphasize the flow lines at the ends the cracks.
An optical microscope with approximately $100 \times$ magnification was set up in a horizontal position on a hydraulic testing machine to permit the observation of tensile specimens during the application of load. The relation between the appearance of craze cracks and flow lines could thus be observed. Of a number of specimens observed in this manner, only three exhibited flow lines. Two of these three specimens broke at strains of approximately 20 percent, which is higher than the usual average strain for this material. The other specimen broke at the usual average value, which is about 9 percent. The specimens crazed at strains of approximately 3 percent as observed in previous tests. The flow lines were first observed at strains of 8 to 10 percent. The lines clearly originated at the ends of craze cracks, forming an X-figure at each end. It was observed that many of the flow lines passed through the ends of several cracks. The growth of craze cracks was not observed after the appearance of the flow markings. This would seem to indicate that crazing may be the mechanism of stress relaxation in these particular cases up to a point, beyond which surface flow becomes the relaxation mechanism.

The network of flow lines noted in the bright-field microscope and shown in figure 15 can also be studied. to advantage with multiple-beam interferometry. When the reference surface and the specimen surface are made as parallel as possible and the filters removed, high dispersion fringes result. For parallel plates, Fizeau fringes yield a uniform tint of given intensity. Local changes in plate separation produce changes in intensity. In the specimen in figure 16 there were residual angles due to surface

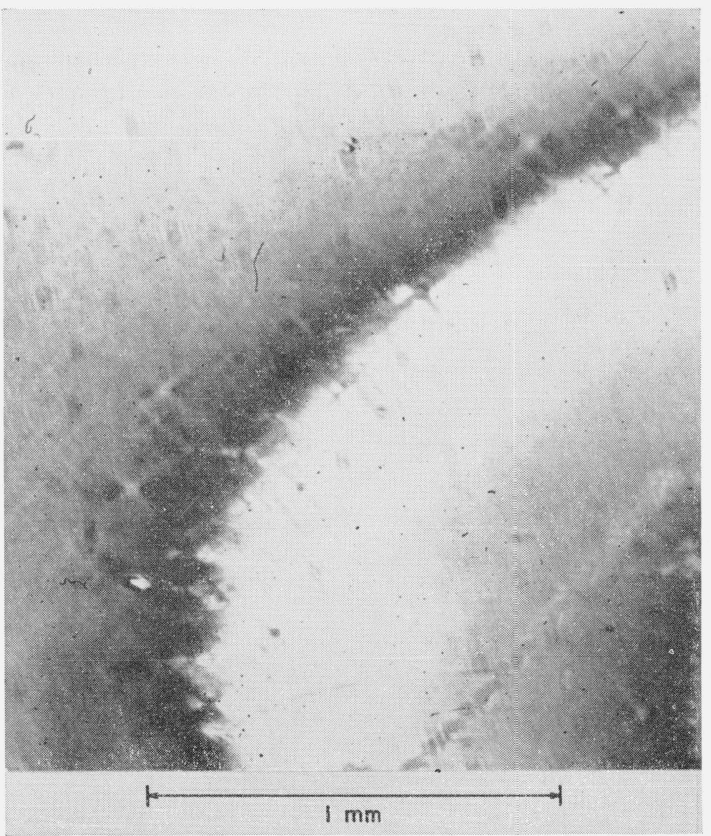

FiguRE 16. Interferogram of a surface of polymethyl methacrylate showing flow at crack nuclei as indicated by changes in intensity.

The reference plate and specimen are nearly parallel. The flow lines are visible as crosses. The broad fringe is a result of body deformation of the specimen. Source is the $546-\mathrm{m} \mu$ line of a mercury lamp. 
features, but over much of the area shown in the photomicrograph, the flow lines are clearly visible as changes in intensity. Tolansky [9] has shown that for a given phase lag and reflectivity a 10-percent change in intensity is equal to $3 \mathrm{~A}$. Although quantitative interpretation of this order would require precise densitometric determinations, which were not attempted in this study, it is obvious that this technique provides a powerful tool for the study of plastic surfaces and of surface structures. However, even a superficial examination revealed that these flow lines involved the surface and permitted a rough approximation of their height above the surface of the order of 25 to $50 \mathrm{~m} \mu$.

Similar flow lines were observed on the surfaces of biaxially stretched acrylic plastic sheet that had been stressed to failure without crazing. In this case, the nuclei consisted of bits of adhesive from the masking paper (fig. 17). Surfaces that craze are also sensitive to the presence of contaminants, such as adhesive, because the most severe crazing and opening up of the cracks is often associated with aggregations of adhesive (fig. 18).

Nadai [18] has shown that flow lines (Luder's lines) are produced by notches or holes. At a sharp reentrant edge the stresses are theoretically infinite, and local yielding in the neighborhood of such discontinuities may result. The resemblance of the flow lines in metal and paraffin specimens illustrated by Nadai bear a marked resemblance to the lines noted in the crazed polymethyl methacrylate tensile specimens. In the case of the uncrazed stretched material shown in figure 17, if the drop of adhesive on the surface is considered as forming a small round area

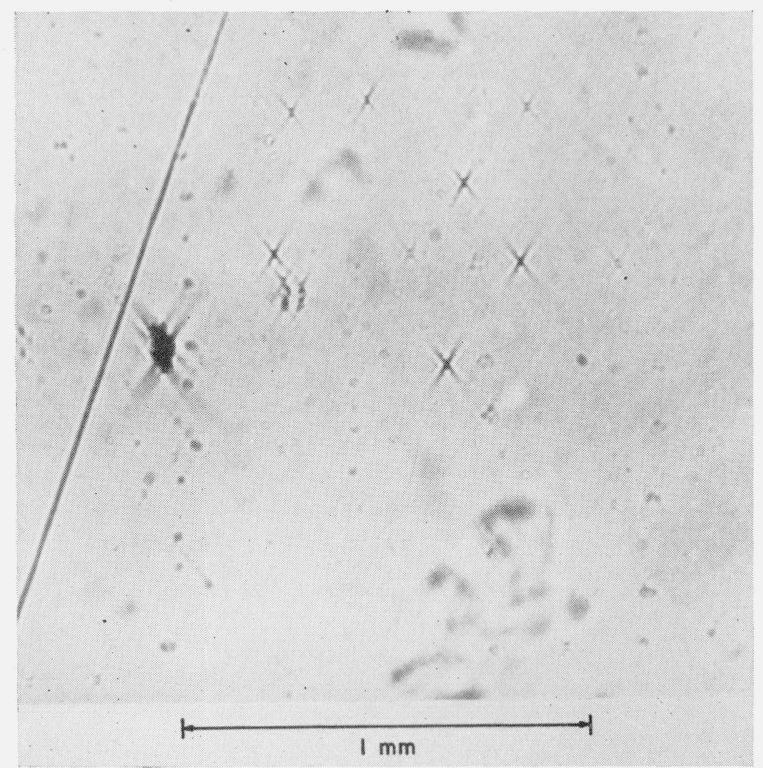

Figure 17. Photomicrograph of the surface of a specimen of biaxially stretched polymethyl alpha-chloroacrylate that has been loaded to failure.

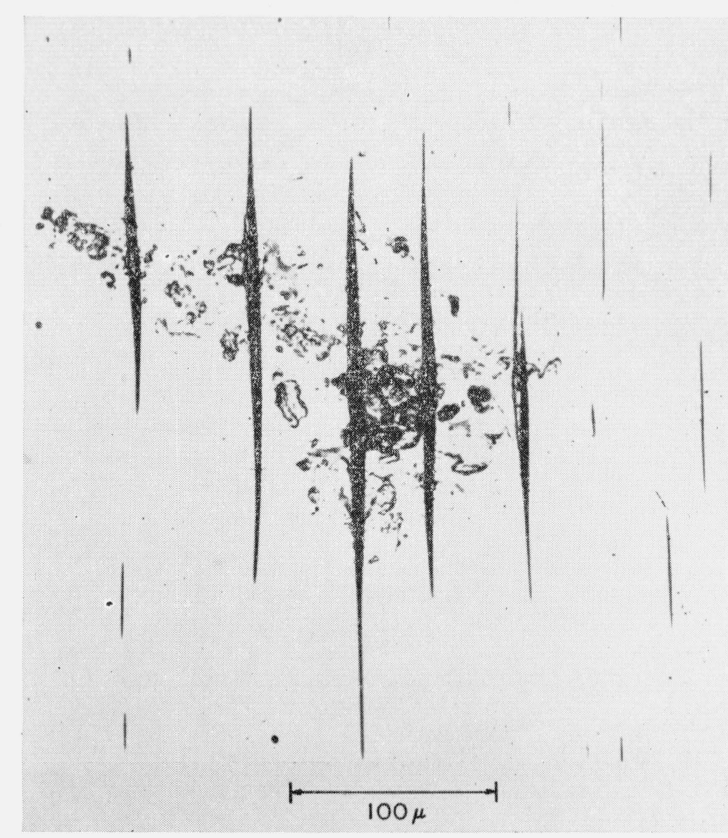

Figure 18. Stress-crazed surface of a sheet of polymethyl methacrylate showing severity of crazing associated with surface contamination by adhesive.

or a small circular hole in the surface film of the sheet, it is also possible to assume a mechanism for the development of the flow lines in these specimens.

For a small hole in a wide plate the tensile stress distribution is well known [19]. Further, the effect of stress concentration is not uniformly distributed around the hole but starts at two points on the boundary of the hole and progresses along a cross-shaped area. From Nadai's exposition it is shown that these two points are the location of peaks of the maximum shear stress on the hole boundary at $\theta=\pi / 2$ and $3 \pi / 2$ and located in a plane perpendicular to the direction of the applied tension. Because this primary yielding is very much localized, it is rarely seen, but as the tensile stress is increased, yielding spreads and soon progresses along two lines symmetrically oriented to the direction of the tensile stress and at an angle of about $45^{\circ}$ with it. The contour lines of the maximum shearing stresses can be checked by photoelastic techniques. Angles of lines observed in the crazed specimens are in fair agreement with those indicated.

In the case of a notch (such as the crazing crack in the surface plane) subjected to tension, much the same result is obtained [18]. F. van Iterson [20] has analyzed the stress situation in a hairline crack across a small part of the section by means of the plastic sector. The solution is also in agreement with the observed angles of the crazing cracks.

The authors acknowledge the assistance of Samuel Levy and Charles Proffer Saylor in the course of this work. 


\section{References}

[1] J. A. Sauer, J. Marin, and C. C. Hsiao, Creep and damping properties of polystyrene, J. Appl. Phys. 20, 507-517 (1949).

[2] B. Maxwell and L. F. Rahm, Rheological properties of polystyrene below 80 C, Ind. Eng. Chem. 41, 1988-1993 (1949).

[3] B. Maxwell and L. F. Rahm, Factors affecting the crazing of polystyrene, J. Soc. Plastics Eng. 6, 7-12 (1950); Tech. Rep. 14B (Plastics Laboratory, Princeton Univ., May 1949).

[4] C. C. Hsiao and J. A. Sauer, On crazing of linear high polymers, J. Appl. Phys. 21, 1071-1083 (1950).

[5] E. W. Russell, Crazing of cast polymethyl methacrylate, Nature 165, 91-96 (1950); Studies on polymethy methacrylate, III: Crazing effects, Rep. Chem. 447 (Royal Aircraft Establishment, Farnborough Hants, England, 1948)

[6] B. M. Axilrod and M. A. Sherman, Effect of stress-solvent crazing on tensile strength of polymethyl methacrylate, Natl. Advisory Comm. Aeronaut., Tech. Note 2444 (1951); Modern Plastics 30, 130-138 (June 1953).

[7] M. A. Sherman and B. M. Axilrod, Stress and strain at onset of crazing of polymethyl methacrylate at various temperatures, Natl. Advisory Comm. Aeronaut., Tech. Note 2778 (1952); Am. Soc. Testing Materials Bul. 191, 65-70 (1953).

[8] J. B. Saunders, Precise topography of optical surfaces, J Research NBS 4\%, 148-155 (1951) RP2239.

[9] S. Tolansky, Multiple beam interferometry of surfaces and films (Clarendon Press, Oxford, England, 1948).
[10] A. Verma, Crystal growth and dislocations (Academic Press, New York, N. Y., and Butterworth Press, London, 1953).

[11] Federal standard stock catalog IV (part 5), Federal specifications for plastics, organic: General specifications, test methods L-P-406b (1952).

[12] S. Dushman, Scientific foundations of vacuum technique (John Wiley \& Sons, Inc., New York, N. Y., 1949)

[13] V. K. Zworykin and E. G. Ramberg, Surface studies with the electron microscope, J. Appl. Phys. 12, 692-695 (1941).

[14] R. W. Ditchburn, Light (Blackie and Son, Ltd., London, 1952).

[15] J. Brossel, Asymmetrical broadening with multiple beam interference fringes, Nature 15\%, 623 (1946).

[16] C. P. Saylor, Accuracy of microscopical methods for determining refractive index by immersion, J. Research NBS 15, 277-294 (1935) RP829.

[17] M. Ross and A. Charlesby, The effect of pile radiation on polymethyl methacrylate (Perspex), I. Atomics 4, 189-194 (1953).

[18] A. Nadai, Theory of flow and fracture of solids (McGrawHill Book Co., Inc., New York, N. Y., 1950).

[19] G. Kirsch, Die Theorie der Elastizität und die Bedürfnisse der Festigkeitslehre, Z. Vereines deut. Ingenieure 4\%, 797 (1898).

[20] F. K. van Iterson, Plasticity in engineering (Hafner Publishing Co., New York, N. Y., 1947).

Washington, December 11, 1956. 